\title{
Factors associated with longitudinal food record compliance in a paediatric cohort study
}

Jimin Yang ${ }^{1, *}$, Kristian F Lynch ${ }^{1}$, Ulla M Uusitalo ${ }^{1}$, Kristina Foterek², Sandra Hummel ${ }^{3}$, Katherine Silvis ${ }^{4}$, Carin Andrén Aronsson ${ }^{5}$, Anne Riikonen ${ }^{6}$, Marian Rewers ${ }^{7}$, Jin-Xiong She ${ }^{4}$, Anette G Ziegler ${ }^{3}$, Olli G Simell ${ }^{8}$, Jorma Toppari ${ }^{9}$, William A Hagopian ${ }^{10}$, Åke Lernmark ${ }^{5}$, Beena Akolkar ${ }^{11}$, Jeffrey P Krischer ${ }^{1}$, Jill M Norris ${ }^{12}$, Suvi M Virtanen ${ }^{13}$,' Suzanne B Johnson ${ }^{14}$ and the TEDDY Study Group $†$

${ }^{1}$ Pediatrics Epidemiology Center, Department of Pediatrics, Morsani College of Medicine, University of South Florida, 3650 Spectrum Blvd, Suite 100, Tampa, FL 33612, USA: ${ }^{2}$ Research Institute of Child Nutrition, Dortmund, Germany: ${ }^{3}$ Institute of Diabetes Research, Helmholtz Zentrum München, and Forschergruppe Diabetes, Klinikum rechts der Isar, Technische Universität München, and Forschergruppe Diabetes e.V., Neuherberg, Germany: ${ }^{4}$ Center for Biotechnology and Genomic Medicine, Medical College of Georgia, Georgia Regents University, Augusta, GA, USA: ${ }^{5}$ Department of Clinical Sciences, Lund University/CRC, Skåne University Hospital SUS, Malmö, Sweden: ${ }^{6}$ National Institute for Health and Welfare, Terveystieteiden yksikkö, Tampereen yliopisto, Finland: ${ }^{3}$ Barbara Davis Center for Childhood Diabetes, University of Colorado School of Medicine, Aurora, CO, USA: ${ }^{8}$ Departments of Pediatrics, Turku University Hospital, Turku, Finland: ${ }^{9}$ Department of Physiology and Pediatrics, University of Turku, Turku, Finland: ${ }^{10}$ Pacific Northwest Diabetes Research Institute, Seattle, WA, USA: ${ }^{11}$ National Institute of Diabetes and Digestive and Kidney Diseases, Bethesda, MD, USA: ${ }^{12}$ Department of Epidemiology, Colorado School of Public Health, Aurora, CO, USA: ${ }^{13} \mathrm{National}$ Institute for Health and Welfare, Nutrition Unit, Helsinki, Finland: ${ }^{14}$ Department of Medical Humanities and Social Sciences, Florida State University College of Medicine, Tallahassee, FL, USA

Submitted 5 December 2014: Final revision received 20 April 2015: Accepted 11 May 2015: First published online 19 June 2015

\begin{abstract}
Objective: Non-compliance with food record submission can induce bias in nutritional epidemiological analysis and make it difficult to draw inference from study findings. We examined the impact of demographic, lifestyle and psychosocial factors on such non-compliance during the first 3 years of participation in a multidisciplinary prospective paediatric study.

Design: The Environmental Determinants of Diabetes in the Young (TEDDY) study collects a $3 \mathrm{~d}$ food record quarterly during the first year of life and semiannually thereafter. High compliance with food record completion was defined as the participating families submitting one or more days of food record at every scheduled clinic visit.

Setting: Three centres in the USA (Colorado, Georgia/Florida and Washington) and three in Europe (Finland, Germany and Sweden).

Subjects: Families who finished the first 3 years of TEDDY participation ( $n$ 8096). Results: High compliance was associated with having a single child, older maternal age, higher maternal education and father responding to study questionnaires. Families showing poor compliance were more likely to be living far from the study centres, from ethnic minority groups, living in a crowded household and not attending clinic visits regularly. Postpartum depression, maternal smoking behaviour and mother working outside the home were also independently associated with poor compliance.

Conclusions: These findings identified specific groups for targeted strategies to encourage completion of food records, thereby reducing potential bias in multidisciplinary collaborative research.
\end{abstract}

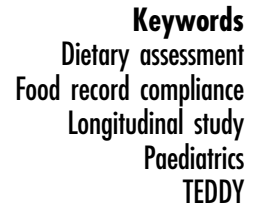

Keywords

ry assessment record compliance
Longitudinal study TEDDY
Food record is a dietary assessment method commonly employed in nutritional epidemiological research.

\footnotetext{
$\dagger$ A full list of the TEDDY Study Group is provided in the online
} supplementary material.
This instrument is able to capture unlimited food choices and is considered the preferred method for populationbased studies, particularly when the participants come from multiple food cultures ${ }^{(1)}$. Since the food record is mostly self-administered, researchers usually provide 
various types of educational materials and tutorials to illustrate how to describe and quantify foods in sufficient detail as well as offer incentives to promote compliance ${ }^{(1)}$.

Despite these efforts, study participants are sometimes non-compliant and fail to submit food records, which may result in selection bias, diminished power to detect associations and serious consequences for interpreting study findings. When the food record method is applied in paediatric populations, the actual work of recording food consumption is mostly performed by parents and nonparental caregivers due to limited cognitive ability of infants and children. Caregivers in day-care and pre-school settings feed many young children at each meal and may not have the time to record intake for a particular child. Parents also feel uncertain when recording meals they did not prepare or observe. These challenges make food records for young children particularly difficult and can lead to non-compliance.

To improve this method of dietary assessment, numerous studies have focused on using digital or mobile devices to generate data of high quality while reducing participant burden ${ }^{(2,3)}$. However, very little has been reported on non-instrument factors impacting the compliance with food record collection, particularly when it is self-administered repeatedly in a longitudinal study. In a study where dietary intake of 0-24-month-old children was evaluated by asking the parents to complete twelve $3 \mathrm{~d}$ weighed food records over two years ${ }^{(4)}$, the participation rate decreased from $90 \%$ when the children were 3 months old to $81 \%$ at 2 years of age, but the reasons for the decreased rate were not discussed.

The Environmental Determinants of Diabetes in the Young (TEDDY) study is a multicentre, multinational, 15-year prospective study of children at high genetic risk of type 1 diabetes (T1D) with the primary goal of identifying environmental triggers of $\mathrm{T}^{\mathrm{D}} \mathrm{D}^{(5)}$. The study protocol includes self-administered questionnaires, self-reported dietary data and biological samples collected repeatedly throughout the study to identify dietary and other environmental factors ${ }^{(5)}$. The objective of the present paper is to examine compliance with food record completion during the first 3 years of TEDDY participation and to identify factors associated with food record compliance.

\section{Methods}

\section{Study design}

The TEDDY study is comprised of six clinical research centres: three in the USA, i.e. Colorado, Georgia/Florida and Washington; and three in Europe, i.e. Finland, Germany and Sweden. Between September 2004 and March 2010, newborn infants from families with firstdegree relatives diagnosed with T1D and from the general populations were asked to participate in HLA genotype screening before the infants turned 4 months of age.
Infants having an illness or birth defect that precluded long-term follow-up or involved use of a treatment that may alter the natural history of diabetes (e.g. steroids or insulin) were excluded from screening. With parental consents obtained pre- or post-delivery, cord blood or heel stick blood samples were collected from 424788 infants to perform genotype screening through either a dried blood spot punch or a small-volume, whole-blood lysate specimen format. If an infant was identified as a carrier of the T1D high-risk HLA genotype, his/her parents were invited to join the follow-up study when the baby was 3.0-4.5 months of age. All parents were fully informed of the infant's increased genetic risk as well as the demands of study participation, which involves clinic visits every 3 months during the first 4 years of the child's life and every 6 months thereafter until the child develops T1D or turns 15 years old. Detailed study design and methods have been published previously ${ }^{(5,6)}$. The study was conducted according to the guidelines laid down in the Declaration of Helsinki. Written informed consents were obtained for all study participants from a parent or primary caregiver, separately, for genetic screening and participation in prospective follow-up. The study was approved by local Institutional Review Boards at every study centre and is monitored by an External Advisory Board formed by the National Institutes of Health.

\section{Dietary assessment procedures}

The TEDDY study employs two dietary assessment methods to collect dietary data, with the food record being the primary one. Dietary intake during the first few months of life mainly consisted of breast milk and infant formula; therefore one $24 \mathrm{~h}$ recall was administered at the initial TEDDY visit (before the child was 4.5 months of age). Trained staff followed the US Department of Agriculture's Automated Multiple-Pass Method (in the USA) ${ }^{(7)}$ or comparable approaches in the TEDDY European countries to collect the $24 \mathrm{~h}$ recall data. As the infant diet became more and more complex, food records were scheduled from 6 months of age to better capture the day-to-day variability in dietary intake.

Previous research suggests the optimal quality of food records is achieved when the recording period lasts 3 to $7 \mathrm{~d}$ and covers weekdays and weekend days ${ }^{(1)}$. A $3 \mathrm{~d}$ recording plan (two weekdays and one weekend day) was adopted in TEDDY considering the demands of other study protocols, which include biological sample collections and detailed recording of the child's illnesses, life stresses and other environmental exposures between clinic visits. Every participating family was instructed to keep a $3 \mathrm{~d}$ record of the child's food consumption at every designated age point (i.e. $6,9,12,18,24,30$ and 36 months of age) and was expected to turn in the records in person or by mail before or at the clinic visit scheduled at every age point.

To facilitate the completion of food records, TEDDY staff provided written instructions and examples on how 
to indicate meal time, meal location, adequate description of foods and beverages, quantity of intake and use of dietary supplements. Based on the Matmallen food portion size booklet developed by the National Food Administration Publishers in Sweden (Livsmedelsverket Publishers), the University of Minnesota's Nutrition Data System for Research (NDS-R) ${ }^{(8)}$ and the participant copy of the Dietary Intervention Study in Children (DISC) Food Amounts Booklet $^{(9)}$, TEDDY developed a food portion size booklet that contained colourful pictorial illustrations of multi-ingredient composite dishes and black-and-white shapes and scales to facilitate portion size estimation. Both the food record form and the booklet were available in the participant's native language and were distributed to the family in person or by mail along with a pre-paid return envelope prior to every scheduled recording period. In Germany, $72 \%$ of the families lived far from the study centre and submitted all of their biological samples and questionnaire data (including the food records) by mail. It was impossible to give them face-to-face instructions on keeping the food records, therefore all German families were asked to keep weighed records in order to achieve protocol consistency within the centre. A $60 \mathrm{ml}$ measuring cup was also offered to the parents to assist with measuring portion size. In the event that food was provided by caregivers other than the parents, the work of keeping food records would be split between parents who had received the food record training and instructions and other caregivers who had not (e.g. day-care staff, relatives, babysitters). These caregivers normally have multiple children in their care, which can make detailed recording challenging. The study-trained family members, in that case, must work with the caregiver(s) to piece together a complete food record for the day. The aforementioned food portion size booklet and measuring cup were also given to the caregivers to help with record keeping.

Multiple quality control procedures were employed in the study to help ensure the quality of food records. The research dietitians and staff in the USA were certified by the University of Minnesota Nutrition Coordinating Center on using the NDS-R program. The dietitians and study nurses in Finland, Germany and Sweden received similar rigorous training. These trained staff reviewed diet records upon receipt to identify missing and ambiguous information and contacted the families as soon as possible to probe for further details. We also observed anecdotally that the parents became familiar with the level of detail required for the food records and they usually would record enough information if they decided to submit a record. On the occasion when the parents or caregivers cannot provide adequate information for a food prepared outside of home, the intake analysis softwares used in the TEDDY countries all have the capacity to handle missing information using default values.

Up to three follow-up contacts (telephone calls or emails) were made to enhance timely submission within the relevant compliance window if a family forgot to return the records by mail or in person at the scheduled TEDDY visit. The food record form was also available in an editable PDF format, permitting caregivers to fill it out on a computer and email it back to the clinics. Gifts and small monetary incentives were offered to promote compliance and they varied across the study centres due to different local Institutional Review Board policies.

\section{Food record compliance}

Considering the possibility that a participant may miss one or more clinic visits and consequently complete none of the study tasks associated with the missed visit(s), we assessed the food record compliance strictly within the completed visits. Specifically, we investigated why participants would not turn in food records given they had attended a clinic visit and had submitted some data for that visit in person, by telephone or by mail. For the purposes of the present study, food record compliance was defined as at least one day's food record submitted during the compliance window associated with a completed clinic visit. There are seven clinic visits scheduled between 6 months and 36 months of age. A compliance window of 3 months around the visit (i.e. from 1.5 months before the visit to 1.5 months after the visit) was created for food records associated with the 6-, 9- and 12-month visits and a compliance window of 6 months was created for food records associated with the 18-, 24-, 30- and 36-month visits. Food record compliance was quantified as the number of visits with at least one day's food record submitted within the compliance window divided by the number of clinic visits completed. A compliance of $100 \%$ means that at least one day's food record was submitted within the compliance window at every completed visit. Families with multiple children enrolled in TEDDY were asked to keep separate records for every participant and their compliance was counted separately.

As of 31 January 2013, 8096 out of the 8677 enrolled infants completed one or more clinic visits between 6 and 36 months of age (Fig. 1, Table 1). Of these participants, seventy-six terminated study participation due to T1D diagnosis per study protocol and 1411 withdrew from the study for a period of time. An additional 209 infants were enrolled but still pending a visit at 36 months. As a result, 4877 scheduled visits could not be completed and no food record was expected. Of the remaining 51795 scheduled visits, 5703 were not completed. Nevertheless a food record was submitted for $439(7.7 \%)$ visits from 411 infants. As the goal was to examine food record compliance among infants actively participating in the study, all these missed visits were excluded. The other 46092 visits were completed and a food record was expected. Food record compliance was examined within this participant group.

\section{Demographic variables}

Child demographic variables included TEDDY study site, sex, whether the child has first-degree relative(s) 


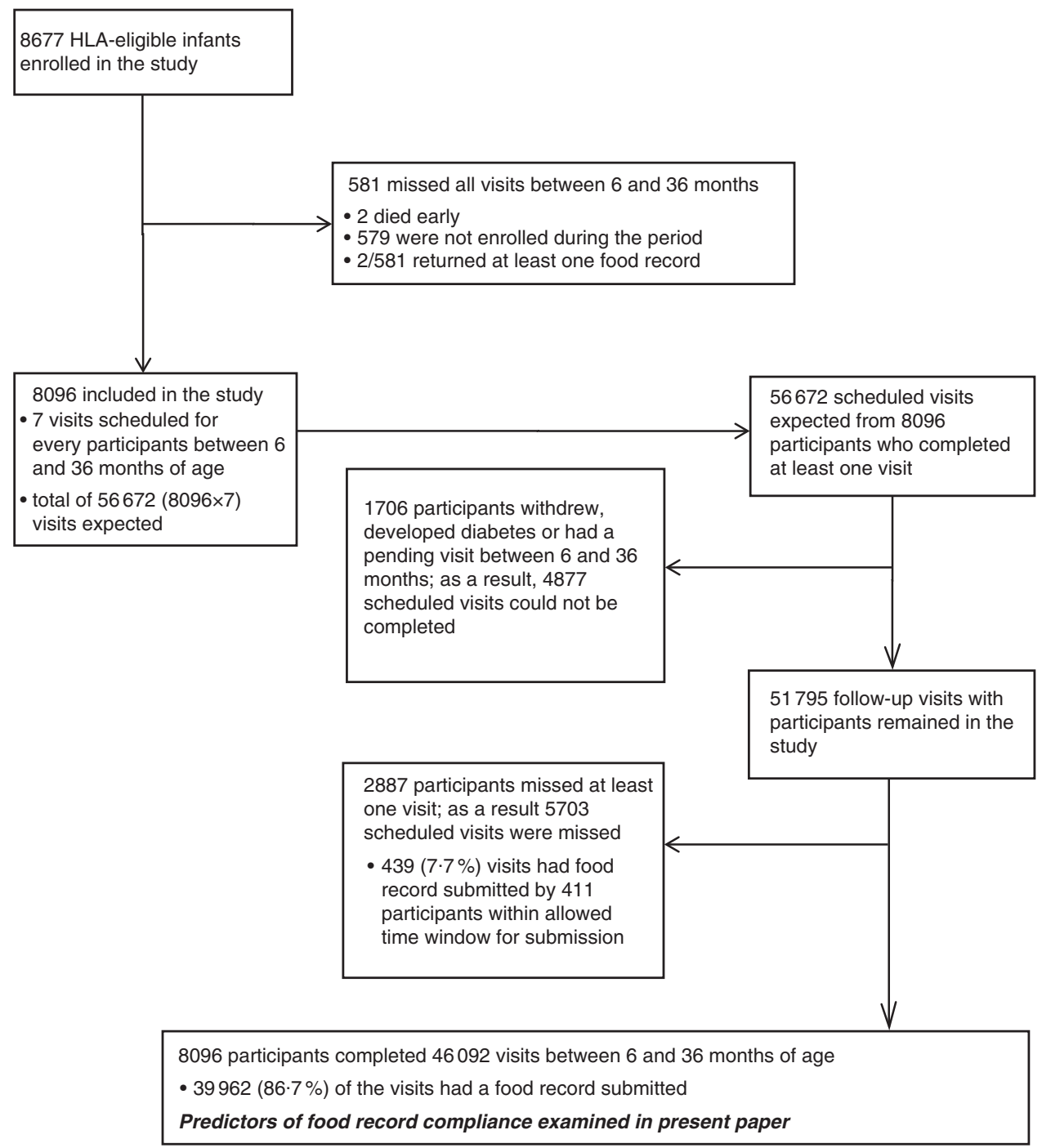

Fig. 1 Participant flow diagram based on the inclusion and exclusion criteria used in the analysis of food record compliance in The Environmental Determinants of Diabetes in the Young (TEDDY) study

(i.e. mother, father and/or sibling) diagnosed with T1D, ethnic minority status and whether the participant is the only child in the family. For US participants, a child was classified as an ethnic minority if his or her mother's first language was not English, or the mother was not born in the USA, or the child was identified by his or her parent as a member of an ethnic minority group based on the US census definition. For participants from Finland, Sweden and Germany, the child was classified as an ethnic minority if the child's mother's first language or country of birth was other than that of the TEDDY country in which the child resided.

Maternal demographic variables included age at time of delivery, marital status (married or living together $v$. single parent) and education (primary education or high school, trade school or some college, graduated from college). Household crowding was measured by the number of persons in the household divided by the number of rooms in the house. Because the crowding variable was skewed, it was re-scored to normalize the distribution $(1=0-0 \cdot 49$; $2=0 \cdot 50-0 \cdot 59 ; 3=0 \cdot 60-0 \cdot 75 ; 4=0 \cdot 76-1 \cdot 00 ; 5=>1 \cdot 00$ ).

\section{Lifestyle variables}

We included three maternal lifestyle behaviours as possible predictors of food record compliance: (i) whether the mother was working outside the home when the child was 9 month of age; (ii) maternal smoking 9 months postpartum; and (iii) maternal consumption of alcohol during the last trimester (alcohol exposure after delivery was not assessed). Child lifestyle factors included whether the child attended day care during the first 3 years of life and if so, the earliest date of starting day care; as well as whether the mother reported placing the child on a special diet due to medical reasons or personal preference.

\section{Maternal psychological status}

Postpartum depression was assessed at the 6-month visit using the Edinburgh Postnatal Depression (EPD) scale 
(coefficient $\alpha=0 \cdot 84)^{(10)}$. We classified mothers as to whether or not their score was above the clinical cut-off $(\geq 13)^{(10)}$. At the same clinic visit, we also assessed maternal anxiety about the child's T1D risk, the accuracy of her perceptions as to the child's risk, whether she was doing something to monitor the child for diabetes onset, whether she had done something to prevent the disease in the child and her satisfaction with study participation. Maternal anxiety about the child's T1D risk was assessed using a six-item measure adapted from the state component of the State-Trait Anxiety Inventory (SAI; coefficient $\alpha=0 \cdot 90)^{(11)}$. If the mother reported the child's T1D risk was higher or much higher than other children's T1D risk, her risk perception was classified as accurate. If she reported the child's T1D risk as the same, somewhat lower or much lower than other children's T1D risk, her risk perception was classified as an underestimate. Study satisfaction was assessed by three questions: 'Overall, how do you feel about having your child participate in the TEDDY study?' (scored: $0=$ 'like it a lot', $1=$ 'like it a little', $2=$ 'it is ok' or 'dislike it'); 'Do you think your child's participation in TEDDY was a good decision?' (scored: $0=$ 'a great decision', 1 = 'a good decision', 2 = 'an ok decision' or 'a bad decision'); and 'Would you recommend the TEDDY study to a friend?' (scored: $0=$ 'yes', $1=$ 'maybe', $2=$ 'no'). The items were significantly correlated and consequently were summed to create a total satisfaction score. Since the total satisfaction scores were not normally distributed and skewed towards 0 , for analysis purposes, we placed each respondent into one of four groups: (i) very satisfied (total score $=0$ ); (ii) satisfied (total score $=1$ or 2); (iii) somewhat satisfied (total score $=3$ or 4); and (iv) neutral/dissatisfied (total score $=5$ or 6 ).

\section{Protocol-related factors}

The protocol-related factors refer to variables describing protocol characteristics that may affect compliance. Because some participants lived very far from the TEDDY clinic, they were placed on a 'long distance protocol' (LDP) which permitted them to collect TEDDY samples at local laboratories and complete other aspects of the protocol by telephone interview and mail. We considered this variable in the analysis because we expected that this protocol characteristic may have been associated with poorer food record compliance. Because families varied in terms of the length of time they had been in TEDDY and their attendance at scheduled TEDDY visits, we also examined whether the number of completed TEDDY visits affected food record compliance. Finally, we tested the association of father's participation in TEDDY with food record compliance by examining whether the TEDDY child's father completed study surveys at enrolment and at the 6-month visit. If he completed both surveys, his participation was scored as 1 . Otherwise, it was scored as 0 .

\section{Statistical analyses}

Food record compliance rates were first described as a function of the number of clinic visits completed. Next, hierarchical multiple regression analysis was applied to identify in blocks the demographic, lifestyle and maternal psychosocial factors that were independently associated with food record compliance measured as a mean percentage of expected food records. We first identified the demographic variables associated with compliance (first block), and then tested for independent association of lifestyle factors (second block) and maternal psychological variables (third block). The fourth block tested the association of protocol-related factors with food record completion. Each block was adjusted for the variables relevant $(P<0 \cdot 1)$ in the prior block(s). A final best association model was selected by backward selection (criterion for removal of variable: $P$ value $<0 \cdot 1)$. The beta coefficient $(\beta)$ describes the change in the mean percentage of expected food records. A negative beta coefficient suggests a lower mean percentage of expected food records submitted at the completed visits. All tests for significance were two-tailed with a significance level of 0.05. Analyses were performed using the statistical software package SAS version $9 \cdot 3$.

\section{Results}

The data in Table 1 represent the characteristics of the 8096 participants included in the current paper. Table 2 depicts food record compliance rates in relation to the number of TEDDY visits completed. The majority of participants returned at least one set of food records, regardless of how many clinic visits were completed. Of the 46092 clinic visits completed by the 8096 participants, $39962(86.7 \%)$ were associated with a returned food record that covered at least one day. Depending on the number of visits completed, the percentage of participants with perfect food record compliance (i.e. submitting food records at all completed visits) varied between $40 \%$ and $71 \%$. From the individual participant's perspective, his or her overall compliance rate over the first 3 years was related to how long the family had been in the study as well as how many scheduled visits the participant actually attended. Such individual overall compliance rate averaged at $84.5 \%$ (SD 24.8\%) among the participants included in the analysis.

We first examined the associations between demographic factors and the food record compliance (first block). There were significant country differences, with Sweden exhibiting the highest compliance rates ( mean $=89.6 \%)$ and Germany $($ mean $=66.9 \%)$ the lowest $(\beta=-21 \cdot 0, P<0 \cdot 001)$. Better compliance was associated with having only one child compared with two or more children $(\beta=+3 \cdot 3, P<0 \cdot 001)$, older maternal age $(\beta=+0.4$ per year, $P<0.001)$, parents married or lived 
Table 1 Characteristics of 8096 TEDDY participants who completed one or more clinic visits between 6 and 36 months of age

\begin{tabular}{|c|c|c|c|c|c|}
\hline & $n$ or mean & $\%$ or SD & & $n$ or mean & $\%$ or SD \\
\hline \multicolumn{3}{|l|}{ Study centre } & \multicolumn{3}{|l|}{ Being the only child } \\
\hline Colorado & 1277 & $15 \cdot 8$ & No & 4412 & 54.5 \\
\hline Georgia/Florida & 867 & $10 \cdot 7$ & Yes & 3150 & 38.9 \\
\hline Washington & 1245 & $15 \cdot 4$ & Missing & 534 & $6 \cdot 6$ \\
\hline Finland & 1760 & $21 \cdot 7$ & Maternal age at child's birth (years) & 30.5 & $5 \cdot 2$ \\
\hline Germany & 565 & $7 \cdot 0$ & Maternal education & & \\
\hline Sweden & 2382 & 29.4 & Higher school or less & 1516 & $18 \cdot 7$ \\
\hline \multicolumn{3}{|c|}{ First-degree relative(s) with type 1 diabetes } & Some college or trade school & 1911 & $23 \cdot 6$ \\
\hline No & 7209 & $89 \cdot 0$ & Graduated from college & 4128 & 51.0 \\
\hline Yes & 887 & 11.0 & Missing & 541 & $6 \cdot 7$ \\
\hline \multicolumn{3}{|l|}{ Child's sex } & \multicolumn{3}{|l|}{ Household crowdingt } \\
\hline Male & 4117 & $50 \cdot 9$ & Normalized score & $2 \cdot 0$ & 1.2 \\
\hline Female & 3979 & $49 \cdot 1$ & & & \\
\hline \multicolumn{6}{|l|}{ Ethnic minority* } \\
\hline No & 6332 & $78 \cdot 2$ & & & \\
\hline Yes & 1255 & $15 \cdot \overline{5}$ & & & \\
\hline Missing & 509 & $6 \cdot 3$ & & & \\
\hline
\end{tabular}

TEDDY, The Environmental Determinants of Diabetes in the Young.

*A child is classified as ethnic minority if his or her mother's first language is not English, or the mother was not born in the USA, or the child is a member of an ethnic minority group based on the US census definition (for US participants); or if the child's mother's first language or country of birth is other than that of the TEDDY country in which the child resides (for participants in Finland, Sweden and Germany).

†Household crowding is evaluated as the number of persons in the household divided by the number of rooms in the house. Because the crowding variable was skewed, it was re-scored to normalize the distribution $(1=0-0.49 ; 2=0.50-0.59 ; 3=0.60-0.75 ; 4=0.76-1.00 ; 5=>1.00)$.

Table 2 Distribution of submitted food records by the total number of clinic visits completed by 8096 TEDDY participants between 6 and 36 months of age

\begin{tabular}{|c|c|c|c|c|c|c|c|c|c|}
\hline \multirow{2}{*}{$\begin{array}{l}\text { Total no. of clinic } \\
\text { visits completed }\end{array}$} & \multirow{2}{*}{$\begin{array}{l}\text { No. of participants with this } \\
\text { no. of visits completed* }\end{array}$} & \multicolumn{8}{|c|}{ Percentage of participants submitting zero, one or more sets of food record(s)† } \\
\hline & & 0 & 1 & 2 & 3 & 4 & 5 & 6 & 7 \\
\hline One & 399 & $29 \cdot 1$ & $70 \cdot 9$ & - & - & - & - & - & \\
\hline Two & 431 & $12 \cdot 8$ & 32.5 & 54.8 & - & - & - & - & - \\
\hline Three & 534 & 4.3 & 14.0 & $24 \cdot 2$ & 57.5 & - & - & - & - \\
\hline Four & 546 & 3.7 & 9.0 & 13.0 & 28.0 & $46 \cdot 3$ & - & - & - \\
\hline Five & 584 & $2 \cdot 1$ & $4 \cdot 1$ & 11.0 & 15.4 & $26 \cdot 9$ & $40 \cdot 6$ & - & - \\
\hline Six & 1089 & $1 \cdot 3$ & $2 \cdot 7$ & 5.5 & 8.2 & $15 \cdot 3$ & $25 \cdot 3$ & 41.8 & - \\
\hline Seven & 4513 & 0.2 & 0.4 & 1.0 & $2 \cdot 4$ & 4.3 & $7 \cdot 2$ & $17 \cdot 3$ & $67 \cdot 1$ \\
\hline
\end{tabular}

TEDDY, The Environmental Determinants of Diabetes in the Young.

${ }^{*}$ Categories by row are mutually exclusive.

†The numbers $0-7$ refer to the total sets of food record(s) submitted between 6 and 36 months of age. At least one clinic visit must be completed before the percentage was calculated.

together compared with other situations $(\beta=+7 \cdot 8$, $P<0.001)$ and higher maternal education compared with high school or less $(\beta=-5.5, P<0 \cdot 001)$. Poorer compliance was associated with ethnic minority status $(\beta=-4 \cdot 1, \quad P<0 \cdot 001)$ and living in a more crowded household $(\beta=-1.4, P<0.001)$. The child's sex was not associated with food record compliance.

We next examined the association of lifestyle behaviours (second block) with food record compliance, controlling for the aforementioned significant demographic factors. Both maternal smoking $(\beta=-5 \cdot 6, P<0 \cdot 001)$ and mother returning to work after the birth of the child (assessed at 9 months, $\beta=-1 \cdot 9, P=0 \cdot 001$ ) were associated with poorer food record compliance. Maternal alcohol consumption during pregnancy, sending the child to day care and the child following a special diet were unrelated to food record compliance.
The next group of factors examined (third block) were indicators of maternal psychological status. Controlling for the relevant demographic and lifestyle factors noted above, mothers with postpartum depression showed poorer food record compliance $(\beta=-4 \cdot 2, P<0 \cdot 001)$ compared with mothers who did not have this condition. Risk perception accuracy, anxiety level, maternal reports of diabetes prevention behaviours and maternal study satisfaction were unrelated to food record compliance.

Protocol-related factors were the final block of variables, controlling for relevant demographic, lifestyle and maternal psychological status variables identified in prior models. The LDP families were found to have poorer food record compliance $(\beta=-5 \cdot 1, P<0 \cdot 001)$ while completing all seven TEDDY visits $(P$ for trend $<0.001)$ and better father participation $(\beta=+6 \cdot 7, P<0 \cdot 001)$ were associated with better food record compliance. 
A final multiple linear regression model was developed by retaining all relevant variables $(P<0 \cdot 1)$ from the previous four blocks (Table 3). Participants from the Georgia/Florida, Colorado and Sweden sites exhibited better compliance than those residing in other TEDDY sites. Being the only child, older maternal age and higher maternal education were also associated with better compliance. Living in a more crowded household and being an ethnic minority were the demographic factors related to poorer compliance. Having one or more first-degree relative(s) with T1D exhibited a significantly lower compliance compared with the rest of the cohort after adjusting for other factors. Among lifestyle factors, mother smoking at 9 months and mother returning to work 9 months after delivery were

Table 3 Final multiple regression model predicting food record compliance during the first 3 years of TEDDY participation ( $n$ 7015)

\begin{tabular}{|c|c|c|c|c|c|}
\hline \multirow[b]{2}{*}{ Factor } & \multicolumn{2}{|c|}{$\begin{array}{l}\text { Percentage of expected food records } \\
\text { completed by the families } \dagger\end{array}$} & \multicolumn{3}{|c|}{ Multiple regression model } \\
\hline & Mean & SD & $\beta$ & $95 \% \mathrm{Cl}$ & $P$ \\
\hline \multicolumn{6}{|l|}{ Study centre } \\
\hline Sweden & $90 \cdot 2$ & 17.4 & 0.0 & Ref. & \\
\hline Colorado & 88.9 & $18 \cdot 6$ & +1.4 & $-0.1,+2 \cdot 9$ & \\
\hline Georgia/Florida & 89.1 & $18 \cdot 2$ & +0.9 & $-0.9,+2 \cdot 6$ & \\
\hline Washington & $81 \cdot 8$ & $22 \cdot \overline{3}$ & -4.9 & $-6 \cdot 5,-3 \cdot 4$ & \\
\hline Finland & 88.6 & $20 \cdot 4$ & -0.9 & $-2 \cdot 1,-0.4$ & \\
\hline Germany & 71.0 & 28.7 & -12.9 & $-15 \cdot 3,-10 \cdot 5$ & $<0.001$ \\
\hline \multicolumn{6}{|c|}{ Having first-degree relative(s) with type 1 diabetes } \\
\hline No & 87.7 & $20 \cdot 2$ & 0.0 & Ref. & \\
\hline Yes & $82 \cdot 7$ & 23.4 & -1.6 & $-3 \cdot 1,-0 \cdot 1$ & 0.03 \\
\hline \multicolumn{6}{|l|}{ Ethnic minority $\ddagger$} \\
\hline No & 88.0 & $19 \cdot 9$ & 0.0 & Ref. & \\
\hline Yes & $82 \cdot 2$ & $23 \cdot 6$ & $-2 \cdot 6$ & $-4 \cdot 0,-1 \cdot 3$ & $<0.001$ \\
\hline \multicolumn{6}{|l|}{ Being the only child } \\
\hline No & $85 \cdot 9$ & 21.4 & 0.0 & Ref. & \\
\hline Yes & 88.7 & $19 \cdot 3$ & $+2 \cdot 4$ & $+1 \cdot 4,+3 \cdot 3$ & $<0.001$ \\
\hline \multicolumn{6}{|l|}{ Maternal age at child's birth } \\
\hline Years & & & +0.3 & $+0.2,+0.4$ & $<0.001$ \\
\hline \multicolumn{6}{|l|}{ Maternal education } \\
\hline High school or less & $83 \cdot 4$ & $23 \cdot 7$ & 0.0 & Ref. & \\
\hline Some college or trade school & 83.5 & $22 \cdot 7$ & $+1 \cdot 7$ & $+0 \cdot 3,+3 \cdot 1$ & \\
\hline Graduated from college & $90 \cdot 1$ & $17 \cdot 7$ & +3.2 & $+1 \cdot 8,+4.5$ & $<0.001$ \\
\hline \multicolumn{6}{|l|}{ Household crowding§ } \\
\hline Norm score & & & $-1 \cdot 0$ & $-1.4,-0.5$ & $<0.001$ \\
\hline \multicolumn{6}{|c|}{ Mother smoked at 9 months postpartum } \\
\hline No & 87.9 & $19 \cdot 9$ & 0.0 & Ref. & \\
\hline Yes & 79.9 & $25 \cdot 0$ & $-3 \cdot 2$ & $-4 \cdot 7,-1 \cdot 7$ & $<0.001$ \\
\hline \multicolumn{6}{|c|}{ Mother worked at 9 months postpartum } \\
\hline No & $87 \cdot 5$ & $20 \cdot 6$ & 0.0 & Ref. & \\
\hline Yes & $86 \cdot 5$ & $20 \cdot 6$ & $-1 \cdot 7$ & $-2 \cdot 7,-0 \cdot 7$ & 0.001 \\
\hline \multicolumn{6}{|l|}{ Postpartum depression at 6 months } \\
\hline No & 87.7 & $20 \cdot 1$ & 0.0 & Ref. & \\
\hline Yes & $81 \cdot 2$ & $24 \cdot 3$ & -3.7 & $-5 \cdot 2,-2 \cdot 1$ & $<0.001$ \\
\hline \multicolumn{6}{|c|}{ Mother reports monitoring the child for diabetes onset } \\
\hline No & 87.8 & $20 \cdot 0$ & 0.0 & Ref. & \\
\hline Yes & $86 \cdot 3$ & $21 \cdot 3$ & -0.9 & $-1 \cdot 8,-0.0$ & 0.05 \\
\hline \multicolumn{6}{|l|}{ Long distance protocol } \\
\hline No & 88.4 & $19 \cdot 3$ & 0.0 & Ref. & \\
\hline Yes & 74.3 & $27 \cdot 0$ & $-5 \cdot 2$ & $-7 \cdot 1,-3 \cdot 3$ & $<0.001$ \\
\hline \multicolumn{6}{|l|}{ Number of clinic visits completed } \\
\hline All seven & $91 \cdot 2$ & $16 \cdot 2$ & 0.0 & Ref. & \\
\hline One or more missed & 80.5 & 24.7 & -7.5 & $-8 \cdot 4,-6 \cdot 6$ & $<0.001$ \\
\hline \multicolumn{6}{|c|}{ Father responded to 3 and 6 month questionnaires } \\
\hline No & 78.6 & $26 \cdot 7$ & 0.0 & Ref. & \\
\hline Yes & 88.2 & $19 \cdot 3$ & $+6 \cdot 7$ & $+5 \cdot 2,+8 \cdot 2$ & $<0.001$ \\
\hline
\end{tabular}

TEDDY, The Environmental Determinants of Diabetes in the Young; Ref., reference category.

Model $R^{2}=0 \cdot 165$

*7015 participants completed at least one clinic visit during the first 3 years of TEDDY participation and had no missing data on the variables analysed in this table.

†Mean (SD) percentage of expected food records completed by the 7015 participants included in the linear regression model.

¥A child is classified as ethnic minority if his or her mother's first language is not English, or the mother was not born in the USA, or the child is a member of an ethnic minority group based on the US census definition (for US participants); or if the child's mother's first language or country of birth is other than that of the TEDDY country in which the child resides (for participants in Finland, Sweden and Germany).

$\S$ Household crowding is evaluated as the number of persons in the household divided by the number of rooms in the house. Because the crowding variable was skewed, it was rescored to normalize the distribution $(1=0-0.49 ; 2=0.50-0.59 ; 3=0.60-0.75 ; 4=0.76-1.00 ; 5=>1.00)$. 
associated with worse compliance. Depression at 6 months postpartum and parental monitoring for diabetes onset were associated with worse compliance. The LDP showed a negative impact, whereas completing more clinic visits and fathers responding to survey questionnaires were positively associated with food record compliance.

\section{Discussion}

Data from more than 8000 young children suggested that the compliance with repeatedly submitting selfadministered $3 \mathrm{~d}$ food records in a prospective observational study was associated with several demographic, lifestyle and psychosocial factors after accounting for overall response to clinic visits. Because food records were kept by caregivers, mostly mothers, during the first 3 years of life, many of the significant factors were maternal or parental characteristics. Given the multidisciplinary nature and the broad range of exposures assessed in the present study, the current paper was focused on examining food record compliance within active study participants who had completed at least one clinic visit between 6 and 36 months of age. The factors associated with overall study compliance and retention, as indicated by clinic visit completion, have been addressed in a separate paper ${ }^{(12)}$.

The difference in food record compliance across study centres was likely related to the different approaches in recording food consumption and the proportion of LDP families at every centre. The lower compliance observed in the German centre reflected these combined challenges, where $72 \%$ participants were LDP families and where this was the only centre that kept weighed food records. The German centre protocol was designed to be country-wide with a focus on children who had a firstdegree relative with T1D, requiring a LDP approach for the majority of families living far from the TEDDY clinic in Munich. These LDP participants completed their clinic visits at their paediatrician's office and turned in biological samples and other data by mail, with follow-up telephone conversations to review data. These constraints presented retention as well as compliance challenges for the German centre. Keeping weighed records is associated with higher burden on the parents and was not always possible if a food was prepared outside of home. Many German parents had indicated that they would rather not complete a food record if they did not know the food weight. This observation agreed with the findings reported in a review article that stated the burden associated with weighing consumed foods increased as children grew older and might lead to under-reporting of dietary intake ${ }^{(13)}$. The proportion of participants on the LDP protocol may also be reflected in the lower compliance observed for the Washington centre (percentage of participants on LDP: Germany $72 \cdot 0 \%$, Finland $2 \cdot 2 \%$, Sweden $0 \cdot 8 \%$, Colorado
$6 \cdot 1 \%$, Georgia/Florida 5.1\%, Washington $17 \cdot 0 \%$ ). This centre has the highest proportion of subjects on LDP $(17 \cdot 0 \%)$ within the USA, reflecting the wider geographic area that was included in the initial screening phase for this centre. Families on the LDP live out of state, or in areas more than an hour from the TEDDY clinic in Seattle are unable to go to one of several satellite clinics that the centre offers. The overall response to study activities and the specific compliance to food records among this group have been consistently lower than in other US sites ${ }^{(14)}$.

The associations between food record compliance and several other demographic factors agreed with previously reported findings on study retention. In the present analysis, older maternal age was found to be an important predictor of better food record compliance, which was consistent with its association with lower study withdrawal in year 1 of TEDDY participation ${ }^{(12)}$. This factor has also been associated with loss to follow-up at 7 years in a prior long-term birth cohort observational study ${ }^{(15)}$. Belonging to an ethnic minority group was associated with high rates of disenrolment ${ }^{(14)}$ and among those enrolled lower compliance with food record collection. The positive associations of higher maternal education level and less crowded household situation with better food record compliance were consistent with their relationship to decreased risk of study withdrawal ${ }^{(12)}$. These two factors may be a proxy for better socio-economic status and a subsequent higher interest in learning how dietary exposure plays a role in T1D disease risk and development, which may have led to better food record compliance. The link between low socio-economic status and study attrition has also been noted in several other long-term observational birth cohort studies ${ }^{(15-18)}$. Having a first-degree relative with T1D showed a weak effect on food record compliance $(P=0.03)$; however, given the size of our study, it may not be relevant at all.

Postpartum depression reported at the 6-month clinic visit as well as smoking and working reported at the 9-month visit may indicate that the mothers were more stressed and had greater difficulty in meeting the demands of TEDDY protocols. Postpartum depression, in particular, was reported at the 6-month visit and showed a strong influence on compliance in the following 24 months, which supported the previous observation that depressed mothers tend to be more unresponsive to tasks rather than withdrawing from studies ${ }^{(19)}$.

Maternal satisfaction with the study and their perception of the child's T1D risk seemed to be irrelevant to the food record compliance. Given these factors were significantly associated with study withdrawal in year $1^{(12)}$ (SB Johnson, unpublished results from the second and third years of TEDDY participation, 2014), the lack of association in the current analysis might be attributed to the fact that food record compliance was evaluated within the context of clinic visits. Father's response to the survey questionnaire at study enrolment and the 6-month visit, on the 
other hand, was consistently associated with lower risk of study withdrawal in year $1^{(12)}$ and better food record compliance.

The lack of association between food record compliance and the practice of special diets suggested that following special diets was not necessarily linked to increased attention on tracking food intake. Many special diets (e.g. gluten free, casein free) involve consuming alternative solid foods, but food choices in the first 3 years of life are relatively limited due to the maturity of the digestive system and, therefore, are likely to be similar between children who followed a special diet and those who did not. As the TEDDY cohort ages, the continued practice of special diets and the efforts spent on providing alternative foods may lead to stronger interest in tracking food intake, which subsequently may boost the compliance with food record collection.

\section{Conclusion}

In conclusion, multiple factors were associated with the compliance with repeated self-administered food records among parents of TEDDY participants. Our analysis addressed the compliance in a situation where parents were aware of their children's elevated risk for T1D when deciding whether or not to continue participation in a multidisciplinary follow-up study that collects data on a broad range of exposures. For some parents, knowing the disease risk may prompt them to pay more attention to feeding practice and tracking their children's intake. Other parents may feel overwhelmed by the effort it takes to describe foods and beverages in adequate detail on top of the other study activities scheduled during and outside clinic visits. The concern of not being able to record food consumption perfectly might have prevented some parents from submitting food records, as they expressed anecdotally that they would not want to start recording if they could not do it well. Such concern may be alleviated by having a staff member assist the parent with recording a partial day or even a whole day of intake over the telephone, which may function as a re-training session to answer questions and reassure parents that they are continuing to complete the task correctly. This approach may be particularly helpful to mothers suffering from postpartum depression and families who are from ethnic minority groups. For the ethnic minority individuals, another strategy that will potentially enhance their compliance is to have a TEDDY staff member who speaks their primary language in addition to the main language of that TEDDY clinic. Other approaches focus on increasing the parents' knowledge of food intake and easing the process of submitting food records. As most of the food records are completed by mothers, some of whom return to work after maternity leave expires, the transition back to work can lead to reduced food record compliance, both because the parents are busier and because the TEDDY participant is in child care. When possible, the parents are encouraged to utilize the meal service information that is already provided by some child-care facilities in conjunction with interviewing the child-care staff to obtain additional details needed for the food record. The option to complete the records online and submit electronically also allows working parents and those living far from the clinics to turn in the record as soon as it is finished, which can reduce lost and forgotten records. These targeted strategies based on our observations may have implications in achieving optimal compliance with self-administered food records in similar multidisciplinary studies initiated shortly after birth. It would be worthwhile to verify our findings in other collaborative projects with individuals facing the same or different disease risks.

\section{Acknowledgements}

Acknowledgements: The authors thank the entire TEDDY study group for their contribution in project development and protocol implementation. The participation of all families is gratefully acknowledged. Financial support: The Environmental Determinants of Diabetes in the Young (TEDDY) study is funded by grants (numbers U01 DK63829, U01 DK63861, U01 DK63821, U01 DK63865, U01 DK63863, U01 DK63836, U01 DK63790, UC4 DK63829, UC4 DK63861, UC4 DK63821, UC4 DK63865, UC4 DK63863, UC4 DK63836 and UC4 DK95300) and a contract (number HHSN267200700014C) from the National Institute of Diabetes and Digestive and Kidney Diseases (NIDDK), the National Institute of Allergy and Infectious Diseases (NIAID), the National Institute of Child Health and Human Development (NICHD), the National Institute of Environmental Health Sciences (NIEHS), the Juvenile Diabetes Research Foundation (JDRF) and the Centers for Disease Control and Prevention (CDC). This work supported in part by the NIH/NCATS Clinical and Translational Science Awards to the University of Florida (grant number UL1 TR000064) and the University of Colorado (grant number UL1 TR001082). The sponsors of this study were represented on the Steering Committee and played a role in the study design, its execution and the plans for publication. The corresponding author had the final decision to submit this manuscript for publication. Conflict of interest: None. Authorship: J.Y. conducted research and wrote the manuscript; K.F.L. analysed data and contributed to the discussion; U.M.U., K.F., S.H., K.S., C.A.A., A.R., J.M.N. and S.M.V. reviewed and edited the manuscript; M.R., J.-X.S., A.G.Z., O.G.S., J.T., W.A.H., A.L., B.A. and J.P.K. designed research and reviewed the manuscript. S.B.J. contributed to the discussion, reviewed and edited the manuscript. Ethics of buman subject participation: This study was conducted according to the guidelines laid down in the Declaration of Helsinki and all 
procedures involving human subjects/patients were approved by local Institutional Review Board at each TEDDY clinical centre and were monitored by an External Evaluation Committee formed by the National Institutes of Health. Written informed consents were obtained from a parent or primary caregiver for both the genetic screening and participation in the prospective follow-up.

\section{Supplementary material}

To view supplementary material for this article, please visit http://dx.doi.org/10.1017/S1368980015001883

\section{References}

1. Willet WC (2013) Nutritional Epidemiology, 3rd ed. New York: Oxford University Press.

2. Stumbo PJ (2013) New technology in dietary assessment: a review of digital methods in improving food record accuracy. Proc Nutr Soc 72, 70-76.

3. Vuckovic N, Ritenbaugh C, Taren DL et al. (2000) A qualitative study of participants' experiences with dietary assessment. I Am Diet Assoc 100, 1023-1028.

4. Verwied-Jorky S, Schiess S, Luque V et al. (2011) Methodology for longitudinal assessment of nutrient intake and dietary habits in early childhood in a transnational multicenter study. J Pediatr Gastroenterol Nutr 52, 96-102.

5. Hagopian WA, Lernmark A, Rewers MJ et al. (2006) TEDDY - The Environmental Determinants of Diabetes in the Young: an observational clinical trial. Ann N Y Acad Sci 1079, 320-326.

6. The TEDDY Study Group (2007) The Environmental Determinants of Diabetes in the Young (TEDDY) study: study design. Pediatr Diabetes 8, 286-298.

7. Raper N, Perloff B, Ingwersen L et al. (2004) An overview of USDA's Dietary Intake Data System. I Food Compost Anal 17, 545-555.
8. Schakel S, Buzzard IM \& Gebhardt SE (1997) Procedures for estimating nutrient values for food composition databases. I Food Compost Anal 10, 102-114.

9. van Horn LV, Stumbo P, Moag-Stahlberg A et al. (1993) The Dietary Intervention Study in Children (DISC): dietary assessment methods for 8- to 10-year-olds. J Am Diet Assoc 93, 1396-1403.

10. Cox JL, Holden JM \& Sagovsky R (1987) Detection of postnatal depression. Development of the 10-item Edinburgh Postnatal Depression Scale. Br J Psychiatry 150, 782-786.

11. Speilberger CD, Gorsuch RL \& Lushene RE (1970) Test Manual for the State-Trait Anxiety Inventory. Palo Alto, CA: Consulting Psychologists Press.

12. Johnson SB, Lee HS, Baxter J et al. (2011) The Environmental Determinants of Diabetes in the Young (TEDDY) study: predictors of early study withdrawal among participants with no family history of type 1 diabetes. Pediatr Diabetes 12, 165-171.

13. Burrows TL, Martin RJ \& Collins CE (2010) A systematic review of the validity of dietary assessment methods in children when compared with the method of doubly labeled water. J Am Diet Assoc 110, 1501-1510.

14. Baxter J, Vehik K, Johnson SB et al. (2012) Differences in recruitment and early retention among ethnic minority participants in a large pediatric cohort: the TEDDY Study. Contemp Clin Trials 33, 633-640.

15. Greene N, Greenland S, Olsen J et al. (2011) Estimating bias from loss to follow-up in the Danish National Birth Cohort. Epidemiology 22, 815-822.

16. Wolke D, Waylen A, Samara M et al. (2009) Selective dropout in longitudinal studies and non-biased prediction of behaviour disorders. Br J Psychiatry 195, 249-256.

17. Kotecha SJ, Watkins WJ, Heron J et al. (2010) Spirometric lung function in school-age children: effect of intrauterine growth retardation and catch-up growth. Am J Respir Crit Care Med 181, 969-974.

18. Howe LD, Tilling K, Galobardes B et al. (2013) Loss to follow-up in cohort studies: bias in estimates of socioeconomic inequalities. Epidemiology 24, 1-9.

19. Dudley J, Jin S, Hoover D et al. (1995) The Multicenter AIDS Cohort Study: retention after 9 1/2 years. Am J Epidemiol 142, 323-330 